\title{
Transoral approach for draining parapharyngeal space abscesses involving multiple maxillofacial spaces
}

\author{
Zhongyan Chen, Jianfeng Liu*, Jianhui Zhao, Jun Han and Dazhang Yang \\ Department of Otolaryngology, China-Japan Friendship Hospital, Beijing, China
}

\begin{abstract}
The parapharyngeal space (PPS) connects multiple maxillofacial spaces (including the submandibular, retropharyngeal, and submasseteric spaces), and it is one of the most complex anatomic locations in the head and neck. Infection in the PPS may cause a series of life-threatening conditions such as mediastinal abscesses. Herein, we present the case of a PPS abscess involving multiple maxillofacial spaces, which was successfully incised and drained using a minimally invasive transoral approach under local anaesthesia. This minimally invasive approach requires full anatomic knowledge of the neck spaces. Additionally, the approach requires that the patient should be able to tolerate local anaesthesia and not have a compromised airway or severe trismus, and the infection should only affect the prestyloid compartment.
\end{abstract}

\section{Introduction}

Although relatively rare, abscesses of the multiple maxillofacial spaces, especially the parapharyngeal space (PPS; also known as the lateral pharyngeal space or pharyngomaxillary space), can progress rapidly, potentially causing life-threatening conditions such as a compromised airway, descending necrotizing mediastinitis, deep vein thrombosis and sepsis [1]. Recent research showed that, in select cases, an uncomplicated deep neck abscess can be effectively treated with antibiotics and careful monitoring, without surgical drainage [2]. Surgical intervention, however, remains the mainstay of treatment for more complicated or severe cases of deep neck infection [3]. Indications for surgery include a compromised airway, critical condition, septicemia, complications, descending infection, diabetes mellitus, and lack of clinical improvement within $48 \mathrm{~h}$ of the initiation of parenteral antibiotics [4-7]. In addition, abscesses greater than $3 \mathrm{~cm}$ in diameter that involve the prevertebral, anterior visceral, or carotid spaces, and those that involve more than two spaces, should be surgically drained [5].

The classic surgical approach for treating a PPS abscess is the transcervical approach. Although this approach can be used to control large blood vessels and nerves, the drainage period is long, and important nerves may be disturbed during the surgery, causing postoperative scars and neurological dysfunction. Recently, minimally invasive ultrasound-guided needle drainage was successfully performed in adult patients [8]. Designing a minimally invasive strategy for draining PPS abscesses is important considering the anatomic complexity, relatively subtle onset, and long hospital stay associated with this condition. Here, we report on the successful drainage of a PPS abscess involving multiple maxillofacial spaces via a transoral approach under local anesthesia combined with antibiotics, which provided an effective conservative alternative treatment strategy. This paper aimed to discuss the indications, safety, and efficacy of this method.

\section{Clinical report}

A 45-year-old female presented with a 4-day history of fever, pharyngalgia, and odynophagia. The patient was transferred from a local hospital where she was diagnosed with acute epiglottis. She had been treated with cephalosporin and dexamethasone for 3 days, but her symptoms were aggravated rather than relieved. The patient was physically healthy and had no comorbidities. On examination, her vital signs were stable, and she did not have a compromised airway. There was swelling and tenderness in the parotid and submandibular area on the right side (Figure 1a) and her skin temperature was high. The size of the mouth opening was limited to two fingers, and observation of the oral cavity and oropharynx revealed bulging and redness in the lateral oropharyngeal wall and soft palate on the right side. Blood tests revealed leukocytosis (white blood cell [WBC] count :17.52 $\times 10^{9} / \mathrm{L}$ ) and elevated C-reactive protein (CRP). Blood culture and pus culture results were all negative. Flexible laryngoscope examination revealed thin suppurative discharges in the lateral pharyngeal wall and the base of the tongue (Figure 1b). Neck and chest computed tomography (CT) scans showed marked inflammation, with abscess formation involving multiple maxillofacial spaces including the PPS, submasseteric space, pterygomandibular space, and submandibular space (Figure 2).

On the second day after hospitalization, the patient's symptoms worsened. An emergency incision and drainage procedure were undertaken via a transoral approach. A transoral vertical incision was made anterior to the palatoglossal arch and posterior to the pterygomandibular fold under local anesthesia with $1 \%$ lidocaine (Figure 3A). After careful dissection of the superior constrictor muscle, a lot of thin fetid purulence oozed out. The abscess cavity was explored with gentle blunt dissection posterolaterally to the PPS, laterally to the pterygomandibular space, and anteroinferiorly to the submandibular space. In addition to draining the PPS, other related spaces exhibiting

${ }^{*}$ Correspondence to: Jianfeng Liu, Department of Otolaryngology, ChinaJapan Friendship Hospital, Beijing, China, Tel: +86 108420 5288; E-mail: mmconfucius@163.com

Key words: parapharyngeal space, abscess, transoral approach, drainage, multiple maxillofacial spaces

Received: September 13, 2019; Accepted: September 27, 2019; Published: September 30, 2019 
suppuration (submasseteric and submandibular spaces) were drained by applying pressure to the masticator and submandibular area. After much irrigation of the abscess cavity with saline, a latex drainage strip

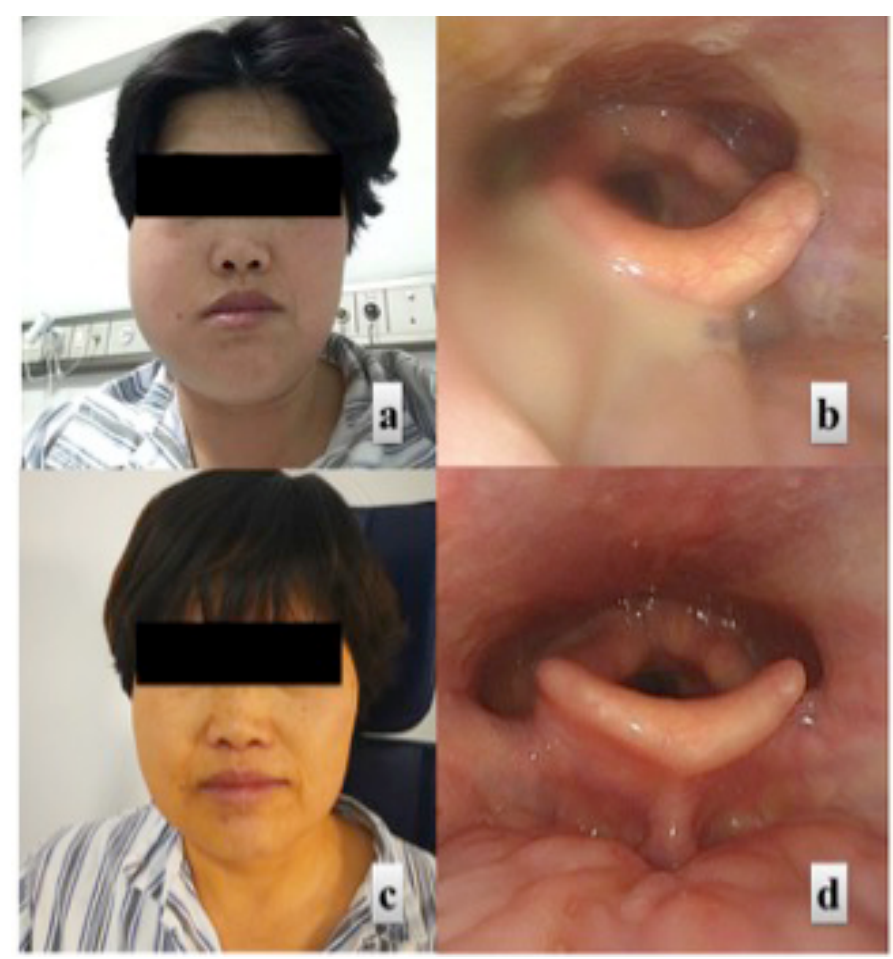

Figure 1. A) The right side of the face is obviously swollen, involving the parotid gland and submandibular area. B) Flexible laryngoscope examination revealed thin suppurative discharges in the lateral pharyngeal wall and the base of the tongue. C) After transoral drainage, the facial swelling disappeared and) the laryngopharynx structure recovered to its normal condition.
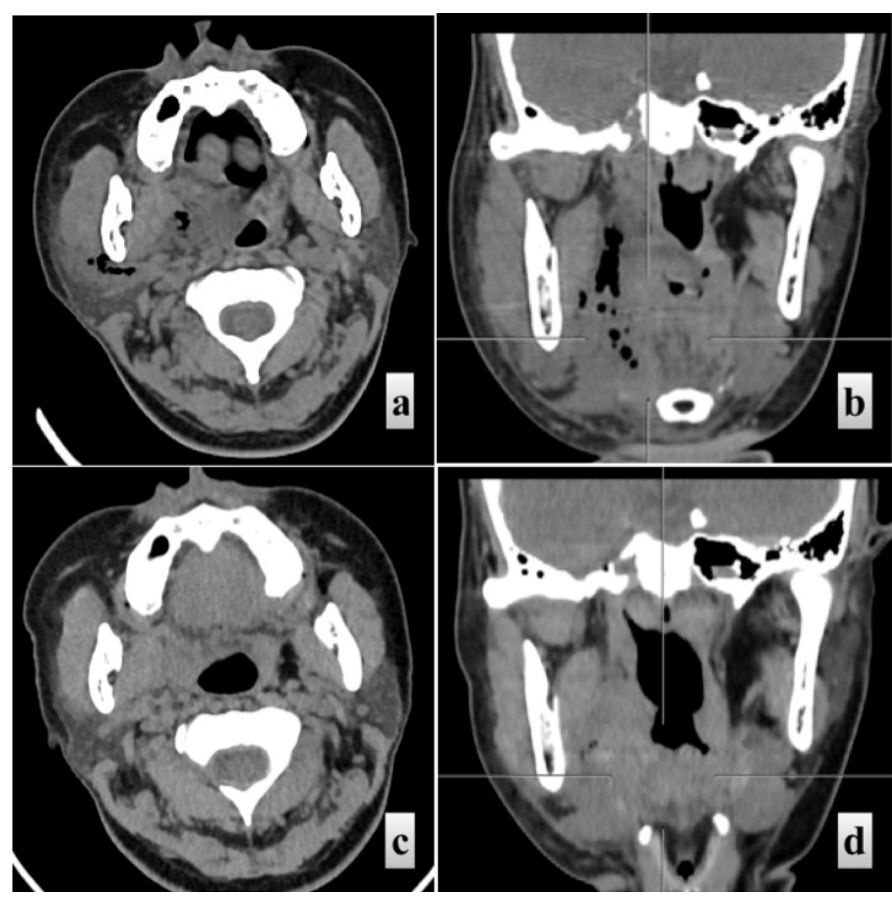

Figure 2. (A) Axial and (B) coronal plain CT scans showing multiple abscesses and air accumulation in the right parapharyngeal space (PPS), submasseteric space, and submandibular space before the treatment. (C) Axial and (D) coronal plain CT scans showing disappearance of abscesses and air 13 days after the treatment.

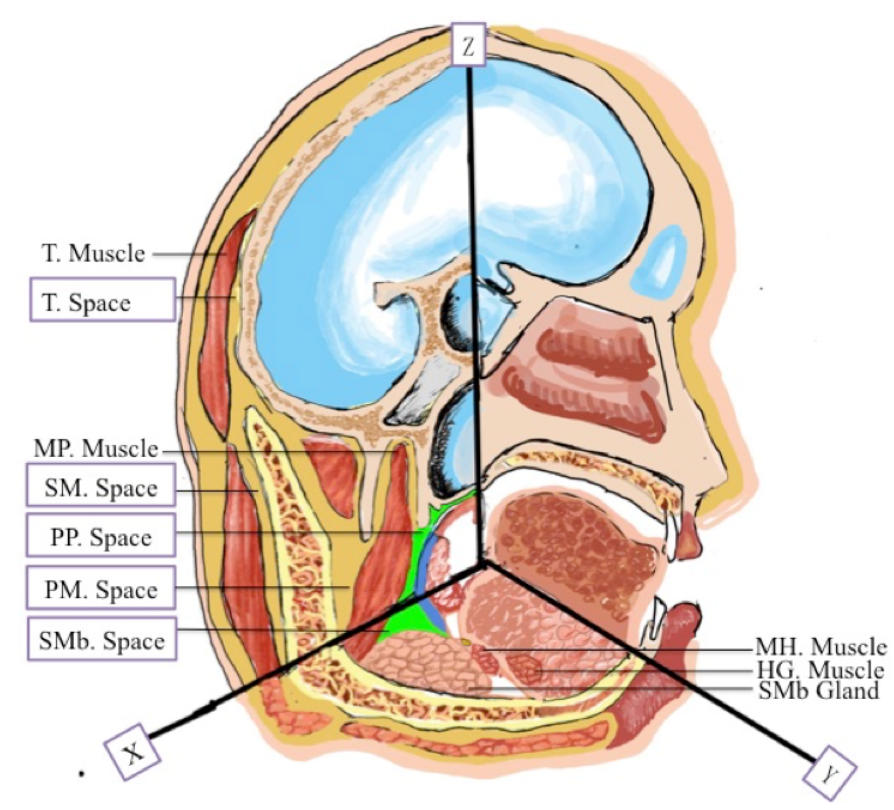

Figure 3. Diagram showing how the PPS communicates with multiple facial and neck spaces in three-dimensional sections. T Space: Temporal Space; MP Muscle: Medial Pterygoid Muscle; SM Space: Submasseteric Space; PP Space: Parapharyngeal Space; PM Space: Pterygomandibular Space; SMb Space: Submandibular Space; MH Muscle: Mylohyoid Muscle; HG Muscle: Hyoglossus Muscle; SMb Gland: Submandibular Gland. $\mathrm{x}-\mathrm{z}$ shows the coronal section through the palatine bone; $\mathrm{x}-\mathrm{y}$ shows the axial section through the submandibular gland; $z-y$ shows the sagittal section through the midline.

was placed deep in the abscess cavity for continuous drainage. The pus was collected for culture and antibiotic sensitivity tests, but the results were all negative. After much irrigation of the abscess cavity with saline, a latex drainage tube was placed deep in the abscess cavity for continuous drainage.

A third-generation cephalosporin (ceftriaxone, $2 \mathrm{~g}$ per day), combined with metronidazole (0.5g per day) was administered intravenously. Additionally, dexamethasone was administered at a dosage of $10 \mathrm{mg}$. The level of CRP and blood count were monitored every 2 days. The patient's symptoms were rapidly relieved day by day, with improvements in both the body temperature and the WBC count. The CT scan was repeated 13 days after the procedure, which revealed that the abscess had disappeared (Figure 2). The patient made a good recovery and was discharged on day 15 after hospitalization (Figure $1 \mathrm{c}$ and $1 \mathrm{~d})$.

\section{Discussion and conclusion}

\section{Anatomy and indications for the approach}

The PPS, which has a pyramidal shape, extends from the sphenoid inferior wall to the hyoid bone. It is divided into two compartments: the prestyloid and poststyloid compartments. The main structure of the poststyloid compartment is the cervical vascular sheath [9]. The incision was made anterior to the palatoglossal arch and slightly posterior to the pterygomandibular fold, that is formed by pterygomandibular raphe, which acted as a window into the PPS [10]. A clamp was inserted between the medial pterygoid muscle and superior constrictor muscle to enable the prestyloid compartment to be reached in order to drain the pus (Figure 3 ). The ventral medial aspect of the medial pterygoid muscle contains the lingual nerve and should be protected. The abscess was limited to the prestyloid compartment far away from the carotid artery, which helped to ensure the feasibility of the procedure. 
Which kinds of patient can be treated using this conservative strategy? First, the airway should not be compromised, which means that a secure airway must be maintained. Before the procedure, flexible laryngoscopy and imaging is necessary to assess the degree of swelling of the lateral pharyngeal wall. Second, the patient should not have severe trismus, as this could lead to poor visualization, limiting the precision of the incision. Third, the patient's cardiopulmonary system should be able to tolerate local anesthesia, so elderly patients, patients with diabetes mellitus, and immunocompromised patients should be excluded. Finally, the infection should only affect the prestyloid compartment, not the prestyloid compartment.

\section{Surgical approaches for treating PPS diseases}

Surgery for dealing with PPS diseases (including both infections and tumors) can be undergone via the external approach and the transoral approach [11]. Minimally invasive approaches for accessing the PPS are a hot topic. Lee et al. [12] first reported endoscopic trans nasal transsphenoidal incision drainage in children with PPS abscesses near skull base. Clear endoscopic identification of anatomical structures not only avoided nerve and blood vessel damage, but also greatly shortened the hospital stay [12]. In addition, researchers have explored the use of a transoral approach combined with endoscopy to remove PPS tumors. In 2013, Iseri et al. [13] reported four cases of PPS tumors (which were mainly found to be pleomorphic adenomas) that were removed by an endoscopic transoral approach. Thereafter, Liu et al. [14] summarized the results of endoscopically assisted transoral resection of a PPS benign tumor. Schwartz et al. [15] reported on a 2-year-old child with a parapharyngeal abscess, located in the inferior wall of the external auditory canal, which was removed through the external auditory canal. Although drainage at the lowest point is often the most appropriate approach, the case reported by Schwartz et al. [16] and the case reported by our team indicate that, in some cases, this is not required, as sufficient drainage can be achieved via assistant extrusion from outside to converge the pus. With the application of $\mathrm{Da}$ Vinci surgical robots, more clinical centers have explored the use of robot-assisted transoral approaches to remove PPS tumors [17].

\section{Controversies regarding the management of deep neck infection}

No consensus has been reached about the optimal management of deep neck infection. Surgical intervention is the mainstay of treatment for deep neck space infection [5]. As some patients have been reported to be cured by using only high-dose intravenous antibiotics and improved imaging techniques, surgical drainage is not always required. A casecontrol study in children showed that for deep neck abscess $>25 \mathrm{~mm}$ the age of children needing surgical drainage was younger than group with antibiotics alone [2]. Age, abscess size, WBC count, and need for intensive care unit admission are useful when judging whether nonsurgical therapy is sufficient. Additionally, cases involving the danger space and those with complications or a compromised airway require surgical intervention [7]. Boscolo-Rizzo et al. [5] suggested that trialing intravenous antibiotic treatment along with a contrast-enhanced CTbased wait-and-watch policy may result in a lower number of patients requiring surgical drainage.
In summary, drainage of PPS abscesses involving multiple maxillofacial spaces via a minimally invasive transoral approach is feasible in selected patients. This method, along with sensitive antibiotics and daily dressing changes, avoids scars made by an external approach, shortens the time of hospitalization, improves the quality of life of the patient, and reduces hospital costs.

\section{References}

1. Alaani A, Griffiths H, Minhas SS, Olliff J, Lee AB (2005) Parapharyngeal abscess: Diagnosis, complications and management in adults. Eur Arch Otorhinolaryngol 262: 345-350. [Crossref]

2. Wong DK, Brown C, Mills N, Spielmann P, Neeff M (2012) To drain or not to drain - management of pediatric deep neck abscesses: A case-control study. Int J Pediatr Otorhinolaryngol 76: 1810-1813. [Crossref]

3. Vieira F, Allen SM, Stocks RM, Thompson JW. (2008) Deep neck infection. Otolaryngol Clin North Am 41: 459-483, vii. [Crossref]

4. Lawrence R, Bateman N (2017) Controversies in the management of deep neck space infection in children: An evidence-based review. Clin Otolaryngol 42: 156-163. [Crossref]

5. Boscolo-Rizzo P, Stellin M, Muzzi E, Mantovani M, Fuson R, et al. (2012) Deep neck infections: A study of 365 cases highlighting recommendations for management and treatment. Eur Arch Otorhinolaryngol 269: 1241-1249. [Crossref]

6. Sichel JY, Dano I, Hocwald E, Biron A, Eliashar R (2002) Nonsurgical management of parapharyngeal space infections: A prospective study. Laryngoscope 112: 906-910. [Crossref]

7. Huppler A, Chun R, Gregory S, Sindhu M (2018) Pediatric deep neck space infections: A medical and surgical perspective. J Pediatr Infectious Dise 14: 043-051.

8. Biron VL, Kurien G, Dziegielewski P, Barber B, Seikaly S (2013) Surgical vs ultrasound-guided drainage of deep neck space abscesses: A randomized controlled trial: Surgical vs ultrasound drainage. J Otolaryngol Head Neck Surg 42: 18. [Crossref]

9. Stambuk HE, Patel SG (2008) Imaging of the parapharyngeal space. Otolaryngol Clin North Am 41: 77-101, vi. [Crossref]

10. Mohamed A, Paleri V, George A (2019) A cadaveric study quantifying the anatomical landmarks of the facial artery and its parapharyngeal branches for safe transoral surgery. Head Neck 41: 3389-3394.

11. Eisele DW, Richmon JD (2013) Contemporary evaluation and management of parapharyngeal space neoplasms. J Laryngol Otol 127: 550-555. [Crossref]

12. Lee CH, Lee TJ, Chen CW (2010) Transnasal endoscopic approach for drainage of pediatric parapharyngeal space abscess. Otolaryngol Head Neck Surg 143: 467-468. [Crossref]

13. Iseri M, Ozturk M, Kara A, et al. (2015) Endoscope-assisted transoral approach to parapharyngeal space tumors. Head Neck 37: 243-248. [Crossref]

14. Liu Y, Yu HJ, Zhen HT (2018) Transoral and endoscope-assisted transoral approaches to resecting benign tumours of the parapharyngeal space located in the medial portion of the carotid sheaths and extending toward the skull base: Our experience. $J$ Laryngol Otol 132: 748-752. [Crossref]

15. Schwartz TR, Colletta MD, McCormick ME (2018) Transcanal incision and drainage of a parapharyngeal abscess in a pediatric patient: A novel technique. Int J Pediatr Otorhinolaryngol 112: 80-81. [Crossref]

16. Chan JY, Tsang RK, Eisele DW, Richmon JD (2015) Transoral robotic surgery of the parapharyngeal space: A case series and systematic review. Head Neck 37: 293-298. [Crossref]

17. Boyce BJ, Curry JM, Luginbuhl A, Cognetti DM (2016) Transoral robotic approach to parapharyngeal space tumors: Case series and technical limitations. Laryngoscope 126: 1776-1782. [Crossref]

Copyright: (C2019 Chen Z. This is an open-access article distributed under the terms of the Creative Commons Attribution License, which permits unrestricted use, distribution, and reproduction in any medium, provided the original author and source are credited. 